\title{
A Summary of Geometric Level-Set Analogues for a General Class of Parametric Active Contour and Surface Models
}

\author{
Chenyang Xu, Johns Hopkins University, chenyang@jhu.edu \\ Anthony Yezzi, Jr., Georgia Institute of Technology, anthony.yezzi@ee.gatech.edu \\ Jerry L. Prince, Johns Hopkins University, prince@jhu.edu
}

\begin{abstract}
Geometric active contours (GACs) and surfaces (GASs) implemented via level set techniques enjoy many advantages over parametric active contours (PACs) and surfaces (PASs), such as computational stability and the ability to change topology during deformation. While many capabilities of earlier PACs and PASs have been reproduced by various GACs and GASs, and while relationships have been discussed for a variety of specific cases, a comprehensive accounting of the connections between these two worlds (particularly regarding rigid forces) has not been consolidated thus far. In this paper we present the precise mathematical relationships between the two for an extensive family of both active contour and surface models, encompassing spatiallyvarying coefficients, both tension and rigidity, and both conservative and non-conservative external forces. The result is a very general geometric formulation for which the intuitive design principles of PACs and PASs can be applied. We also point out which type of PAC and PAS methodologies cannot be adapted to the geometric level set framework. We conclude by demonstrating several geometric adaptations of specific PACs and PASs in several simulations.
\end{abstract}

\section{Introduction}

Active contours are curves that deform within digital images to recover object shapes [14]. They are classified as either parametric active contours (PACs) (cf. $[12,14])$ or geometric active contours (GACs) (cf. [5, 6, 17, 41]) according to their representation and implementation. In particular, PACs are represented explicitly as parameterized curves $[12,14]$ in a Lagrangian formulation. GACs are represented implicitly as level sets of two-dimensional distance functions $[3,16,30]$ which evolve according to an Eulerian formulation. They are based on the theory of curve evolution implemented via level set techniques [23].

PACs are the older of the two formulations and have been used extensively in many applications over the last decade (see [19], for example). A rich variety of modifications based on physical and non-physical concepts have been implemented to solve different shape estimation problems $[12,34,39]$. GACs were introduced more recently and were hailed as the solution to the problem of required topological changes during curve evolution $[5,17]$. Modifications and enhancements have been added to change their behavior or improve their performance in a variety of applications $[6,15,33,41]$, including a number of regionbased models which have appeared recently in the literature [9, 24, 25, 29, 42, 43].

While similarities between the two formulations have always been apparent, only recently have the precise relationships begun to emerge in the literature. Caselles et al. [6] showed that their GACs are equivalent to a special class of classical PACs. Aubert and Blanc-Féraud [4] revisited this equivalence and extended it to the 3-D (active surface) case. The equivalence derived in these two cases is limited in two respects, however. First, it applies only to those active contours derived from energy minimization principles. Thus, the question of whether a geometric formulation can be found for more general active contours is not addressed. Second, the equivalence only applies to active contours with elastic forces; rigid forces are neglected. Whitaker et al. [36] included rigid forces in their formulation, but only with constant weights and conservative forces.

Overall, the equivalences currently established in the literature do not relate a full family of parametric models to their geometric equivalent. As a result, it is difficult to design GACs that take advantage of the wealth of parametric models that have been previously established. For example, it is not clear how one would incorporate non-conservative external forces, such as the forces defined in [38,39]. Also, it is not clear how to incorporate regional pressure forces, such as those used in $[27,28]$, although specialized regionbased GACs [9, 24, 29, 43] have been proposed recently. Meanwhile, it has been well known that the use of elastic internal forces may cause undesirable shrinking effect, whereas the use of rigid internal forces can smooth the contour without this adverse effect. However, the use of rigid internal forces have been largely lacking in GAC formulations so far. Since these are commonly used features in PACs, there is a clear need to establish an equivalent model in GACs so that the computational and topological advantages of GACs can be simultaneously exploited.

In this paper, we derive an explicit mathematical relationship between the general formulations of PACs and GACs. The formulation considered here allows both conservative and non-conservative external force as well as both elastic and rigid internal forces with spatially-varying weights. This equivalence relationship allows straightforward translation from almost any PAC to a GAC and vice 
versa. An extension of this equivalence to active surfaces for volumetric image segmentation is presented as well. The proposed equivalence does not directly apply, however, to forces that are intrinsically Lagrangian, such as the usual external spring forces or variable tension and rigidity defined on a curve parameterization. We show examples demonstrating how the more general formulation of GACs and GASs can be used to good advantage by exploiting tricks from the PAC and PAS literature. Note that a preliminary version of this paper, which covers the $2 \mathrm{D}$ case, can be found in [40].

\section{Background}

\subsection{Parametric active contours}

The classical PACs, proposed by Kass et al. [14], are formulated by minimizing an energy functional that takes a minimum when contours are smooth and reside on object boundaries. Solving the energy minimization problem leads to a dynamic equation that has both internal and external forces. The external forces resulting from this formulation are conservative forces in that they can be written as gradients of scalar potential functions. Active contours using non-conservative forces, however, have been shown to have improved performance over traditional energy-minimizing active contours [38,39]. Therefore, we now formulate PACs directly from Newton's law, which permits use of the most general external forces.

Mathematically, a PAC is a time-varying curve $\boldsymbol{X}(s, t)=[X(s, t), Y(s, t)]$ where $s \in[0,1]$ is arclength and $t \in \mathbf{R}^{+}$is time. The dynamics of the curve are governed by the equation

$$
\gamma \boldsymbol{X}_{t}=\boldsymbol{F}_{\text {int }}+\boldsymbol{F}_{\text {ext }}
$$

where $\boldsymbol{X}_{t}$ is the partial derivative of $\boldsymbol{X}$ with respect to $t$, $-\gamma \boldsymbol{X}_{t}$ is the damping force with $\gamma$ being an arbitrary nonnegative constant, and $\boldsymbol{F}_{\text {int }}$ and $\boldsymbol{F}_{\text {ext }}$ are internal and external forces, respectively. The contour comes to a rest when the net effect of the damping, internal, and external forces reaches zero. The external force is designed to pull an active contour towards object boundaries or other features of interest. Many types of external forces have been developed in the past (see [37] for a comprehensive list of external forces), including the well-known pressure force [12] and the Gaussian potential force [14]. The internal force is the sum of elastic and rigid forces defined as follows

$$
\begin{aligned}
\boldsymbol{F}_{\text {elastic }} & =\left[\alpha(s, t) \boldsymbol{X}_{s}(s, t)\right]_{s} \\
\boldsymbol{F}_{\text {rigid }} & =-\left[\beta(s, t) \boldsymbol{X}_{s s}(s, t)\right]_{s s},
\end{aligned}
$$

where the coefficients $\alpha(s, t)$ and $\beta(s, t)$ can be used to control the strength of the contour's elasticity and rigidity, respectively. In this general formulation, these coefficients are allowed to vary both along the length of the curve and over time. In practice, $\alpha$ is usually a positive constant and $\beta$ is usually zero. It is important to maintain the most general formulation, however, in order to understand the precise relationship between PACs and GACs.

\subsection{Geometric active contours}

GACs $[5,17]$ are based on the theory of curve evolution $[3,16,30]$ and the level set method [23]. In this framework, curves evolve using only geometric measures, resulting in a contour evolution that is independent of the curve's parameterization. This avoids the need to repeatedly reparameterize the curve or to explicitly handle topological changes (cf. [18]). The parametric representations of the curves themselves are computed only after the evolution of the level set function is complete.

Let $\phi(x, t)$ be a 2-D scalar function whose zero level set defines the GAC. Osher and Sethian [23] have shown that if a curve evolution is given by

$$
\boldsymbol{X}_{t}=F(\kappa) \boldsymbol{N}
$$

where $\boldsymbol{N}$ is the inward unit normal and $F(\kappa)$ is a function of curvature $\kappa$, then its evolution can be exactly simulated by evolving $\phi(\boldsymbol{x}, t)$ according to the dynamic equation

$$
\phi_{t}=F(\kappa)|\nabla \phi|
$$

assuming the initial curve and the initial zero level set coincide. We refer this result as the fundamental relationship of level set evolution, which provides the basis for all the level set evolution work thereafter.

The original GAC formulation $[5,17]$ is given by

$$
\phi_{t}=c\left(\kappa+V_{0}\right)|\nabla \phi|,
$$

where $\kappa=\nabla \cdot(\nabla \phi /|\nabla \phi|)$ is the curvature, $V_{0}$ is a constant, and $c \equiv c(\boldsymbol{x})=\left(1+\left|\nabla\left(G_{\sigma}(\boldsymbol{x}) * I(\boldsymbol{x})\right)\right|\right)^{-1}$ is an edge potential derived from the image. In (6), the product $c(\kappa+$ $V_{0}$ ) determines the overall evolution speed of level sets of $\phi(\boldsymbol{x}, t)$ along their normal direction.

This scheme works well for objects that have good contrast. When the object boundary is indistinct or has gaps, however, the contour tends to leak through the boundary. To address this problem, $[6,15,41]$ propose the following

$$
\phi_{t}=c\left(\kappa+V_{0}\right)|\nabla \phi|+\nabla c \cdot \nabla \phi .
$$

The extra stopping term $\nabla c \cdot \nabla \phi$ is used to pull back the contour if it passes the boundary. To further reduce the sensitivity to boundary leaking problem, [33] enhanced the above formulation with

$$
\phi_{t}=(c \kappa|\nabla \phi|+\nabla c \cdot \nabla \phi)+V_{0}\left(c+\frac{1}{2} x \cdot \nabla c\right)|\nabla \phi|
$$

by adding an extra term $V_{0} \frac{1}{2} \boldsymbol{x} \cdot \nabla c|\nabla \phi|$ to provide more stopping power at boundaries.

It has been observed that even (8) does not provide a satisfactory solution to the boundary leaking problem; contours can still leak through boundary gaps and weak edges. 
Meanwhile, several good solutions to this problem have appeared in recent PAC literature [27, 28, 39]. But it is not clear, however, how these solutions can be adapted to the GAC framework. In this paper, we make this connection explicit by deriving a mathematical relationship between PACs and GACs and their extensions to active surfaces. We then show how two new GACs can be obtained through this relationship, and we demonstrate how the boundary leaking problem is solved using these new active contour models.

\section{Relationship between parametric and geo- metric active contours}

In this section, we introduce a general formulation for GACs by reformulating (1) using a level set representation. We start by modifying the parametric problem according to the following two considerations. First, we separate pressure forces $\boldsymbol{F}_{\text {pres }}(\boldsymbol{X})$ from other external forces $\boldsymbol{F}_{\text {ext }}(\boldsymbol{X})$ because they require a special numerical schemes [23]. Second, we consider only the normal component of force since the tangential component affects an active contour's parameterization but not its geometry. Therefore, the class of parametric models we consider is given by

$$
\gamma \boldsymbol{X}_{t}=\left[\left(\boldsymbol{F}_{\mathrm{int}}+\boldsymbol{F}_{\mathrm{pres}}+\boldsymbol{F}_{\mathrm{ext}}\right) \cdot \boldsymbol{N}\right] \boldsymbol{N}
$$

where $\boldsymbol{N}$ is the inward unit normal. The pressure force is given by $\boldsymbol{F}_{\text {pres }}=w_{\text {pres }}(s, t) \boldsymbol{N}$, which generalizes Cohen's balloon force [12] by allowing the weight to be spatially and temporally varying.

\subsection{Equivalence}

Using the fundamental relationship of level set evolution given by (4) and (5), as well as differential geometry properties of planar curves, it can be shown that a general formula for a GAC mimicking the PAC given by (9) can be written as follows (details of the derivation can be found in our previous paper [40]):

$$
\begin{gathered}
\gamma \phi_{t}(\boldsymbol{x})=\left[\alpha(\boldsymbol{x}) \kappa(\boldsymbol{x})+\beta(\boldsymbol{x}) \kappa^{3}(\boldsymbol{x})-\rho(\boldsymbol{x})\right]|\nabla \phi(\boldsymbol{x})| \\
+w_{\text {pres }}(\boldsymbol{x})|\nabla \phi(\boldsymbol{x})|-\boldsymbol{F}_{\text {ext }}(\boldsymbol{x}) \cdot \nabla \phi(\boldsymbol{x}),
\end{gathered}
$$

where

$$
\rho(\boldsymbol{x})=\frac{\hat{\kappa}_{x x} \phi_{y}^{2}+\hat{\kappa}_{y y} \phi_{x}^{2}-2 \hat{\kappa}_{x y} \phi_{x} \phi_{y}}{\phi_{x}^{2}+\phi_{y}^{2}}-\kappa \frac{\hat{\kappa}_{x} \phi_{x}+\hat{\kappa}_{y} \phi_{y}}{\left(\phi_{x}^{2}+\phi_{y}^{2}\right)^{1 / 2}}
$$

and $\hat{\kappa}(\boldsymbol{x})=\beta(\boldsymbol{x}) \kappa(\boldsymbol{x})$. Note that $\alpha(\boldsymbol{x}) \kappa(\boldsymbol{x})$ and $\beta(\boldsymbol{x}) \kappa^{3}(\boldsymbol{x})-\rho(\boldsymbol{x})$ are derived from elastic and rigid internal forces, respectively.

Equation (10) gives a general GAC formula rigorously tied to the standard PAC formulation, and provides a common basis for comparing various GAC formulations and designing new ones. For example, table 1 compares the three GAC formulations described in section 2.2.

\begin{tabular}{c|c|cccc}
\hline Authors & Eq. & $\alpha(\boldsymbol{x})$ & $\beta(\boldsymbol{x})$ & $w_{\text {pres }}(\boldsymbol{x})$ & $F_{\text {ext }}(\boldsymbol{x})$ \\
\hline$[5,17]$ & $(6)$ & $c(\boldsymbol{x})$ & 0 & $c(\boldsymbol{x}) V_{0}$ & 0 \\
{$[6,15,41]$} & $(7)$ & $c(\boldsymbol{x})$ & 0 & $c(\boldsymbol{x}) V_{0}$ & $-\nabla c(\boldsymbol{x})$ \\
{$[33]$} & $(8)$ & $c(\boldsymbol{x})$ & 0 & $V_{0}[c(\boldsymbol{x})+$ & $-\nabla c(\boldsymbol{x})$ \\
& & & & $\left.\frac{1}{2} \boldsymbol{x} \cdot \nabla c(\boldsymbol{x})\right]$ & \\
\hline
\end{tabular}

Table 1. Comparison of existing GACs using (10).

It is apparent from this comparison that previous creativity in the development of new GACs has been somewhat limited. For example, pressure forces have typically been tied through $c(\boldsymbol{x})$, the edge potential, to internal tension forces. External forces have been limited to irrotational forces, and these also tied to $c(\boldsymbol{x})$. Finally, the use of rigid forces has been largely missing in existing GACs. There is room for much creativity in the selection of parameters for a GAC, and the derived equivalence should help to identify the opportunities and their relation to PACs. We explore some possibilities in the Applications section.

\section{Active Surface Extensions}

In this section, we study the general relationship between parametric and geometric active surfaces (PASs and GASs). Like active contours, the class of PASs we consider obeys similar dynamic equation as (9). Given a parametric surface, $\boldsymbol{X}(u, v)=[x(u, v), y(u, v), z(u, v)]^{T},(u, v) \in$ $[0,1] \times[0,1]$, the classes of internal forces we concern minimizes the following deformation energies

$$
\begin{gathered}
E_{\text {elastic }}=\int \frac{\alpha(u, v)}{2}\left(\left|\boldsymbol{X}_{u}\right|^{2}+\left|\boldsymbol{X}_{v}\right|^{2}\right) d u d v \\
E_{\text {rigid }}=\int \frac{\beta(u, v)}{2}\left(\left|\boldsymbol{X}_{u u}\right|^{2}+2\left|\boldsymbol{X}_{u v}\right|^{2}+\left|\boldsymbol{X}_{v v}\right|^{2}\right) d u d v
\end{gathered}
$$

where $\boldsymbol{X}_{u}, \boldsymbol{X}_{v}, \boldsymbol{X}_{u u}, \boldsymbol{X}_{u v}$, and $\boldsymbol{X}_{v v}$ denote the surface's first and second partial derivatives, and $E_{\text {elastic }}$ and $E_{\text {rigid }}$ are the well-known membrane and thin-plate functionals [35]. Taking the variational derivatives of deformation energies yields the corresponding elastic and rigid forces

$$
\begin{aligned}
\boldsymbol{F}_{\text {elastic }} & =\tilde{\nabla} \cdot[\alpha(u, v) \tilde{\nabla} \boldsymbol{X}] \\
\boldsymbol{F}_{\text {rigid }} & =-\tilde{\triangle}[\beta(u, v) \tilde{\triangle} \boldsymbol{X}]
\end{aligned}
$$

where $\tilde{\nabla}=\left(\frac{\partial}{\partial u}, \frac{\partial}{\partial v}\right)^{T}$ and $\tilde{\triangle}=\frac{\partial^{2}}{\partial u^{2}}+\frac{\partial^{2}}{\partial v^{2}}$ denote the gradient and the Laplacian operators defined in the parametric space. Note that in this paper, the typical Euclidean gradient and Laplacian operators in $\boldsymbol{R}^{3}$ are denoted as $\nabla$ and $\triangle$, respectively. Note also that if $\alpha(u, v)$ and $\beta(u, v)$ are set to constants, the elastic force and the rigid force reduces to the Laplacian and the biharmonic (evaluated in parametric space) of the surface (cf. [20]).

A GAS evolves according to geometric properties independent of the surface parameterization. We can therefore assume the surface is parameterized using arc-length 
along principal directions ${ }^{1}$. Some differential geometry notations (for details, see [13]) used in this section are:

- the coefficients of the first fundamental form:

$$
E=\boldsymbol{X}_{u} \cdot \boldsymbol{X}_{u}, F=\boldsymbol{X}_{u} \cdot \boldsymbol{X}_{v}, G=\boldsymbol{X}_{v} \cdot \boldsymbol{X}_{v}
$$

- the coefficients of the second fundamental form:

$$
e=\boldsymbol{X}_{u u} \cdot \boldsymbol{N}, f=\boldsymbol{X}_{u v} \cdot \boldsymbol{N}, g=\boldsymbol{X}_{v v} \cdot \boldsymbol{N}
$$

where $\boldsymbol{N}=\boldsymbol{X}_{u} \times \boldsymbol{X}_{v} /\left|\boldsymbol{X}_{u} \times \boldsymbol{X}_{v}\right|$ is the unit normal.

- mean curvature: $H=\frac{e G-2 f F+g E}{2\left(E G-F^{2}\right)}$, and

Gaussian curvature: $K=\frac{e g-f^{2}}{E G-F^{2}}$.

For a surface parameterized by arc-length along the principal directions, we have $E=1, F=0, G=1$, and $f=0$. Thus, mean curvature and Gaussian curvature take the following simple form

$$
K=e g, \quad H=(e+g) / 2 \text {. }
$$

As in the 2D case, let us first examine the normal component of the elastic force

$$
\begin{aligned}
& \boldsymbol{F}_{\text {elastic }}(\boldsymbol{X}(u, v)) \cdot \boldsymbol{N}=\tilde{\nabla} \cdot[\alpha(u, v) \tilde{\nabla} \boldsymbol{X}] \cdot \boldsymbol{N} \\
& =\left(\alpha_{u}(u, v) \boldsymbol{X}_{u}+\alpha_{v}(u, v) \boldsymbol{X}_{v}+\alpha(u, v) \tilde{\triangle} \boldsymbol{X}\right) \cdot \boldsymbol{N}
\end{aligned}
$$

Since $\boldsymbol{X}_{u} \cdot \boldsymbol{N}=0$ and $\boldsymbol{X}_{v} \cdot \boldsymbol{N}=0$, we have

$$
\boldsymbol{F}_{\text {elastic }}(\boldsymbol{X}(u, v)) \cdot \boldsymbol{N}=\alpha(u, v) \tilde{\triangle} \boldsymbol{X} \cdot \boldsymbol{N}
$$

Note that equation (15) appears the same as if one starts with a constant $\alpha$ and then just replaces it with a spatiallyvarying $\alpha(u, v)$. However, the real reason for $\alpha(u, v)$ coming out of the divergence operator is because the terms consisting of derivatives of $\alpha(u, v)$ are in the tangent space and thus eliminated by the inner product with the normal.

Using (14), we can further simplify the normal component of the elastic force to

$$
\boldsymbol{F}_{\text {elastic }}(\boldsymbol{X}(u, v)) \cdot \boldsymbol{N}=2 \alpha(u, v) H .
$$

Hence, the equivalent level set representation of elastic force can be expressed as

$$
\boldsymbol{F}_{\text {elastic }}(\boldsymbol{x})=2 \alpha(\boldsymbol{x}) H,
$$

where $H$ is given by $\nabla \cdot(\nabla \phi /|\nabla \phi|)$.

For the normal component of the rigid force, we have

$$
\begin{aligned}
& \boldsymbol{F}_{\text {rigid }}(\boldsymbol{X}(u, v)) \cdot \boldsymbol{N}=-\tilde{\triangle}[\beta(u, v) \tilde{\triangle} \boldsymbol{X}] \cdot \boldsymbol{N} \\
& =-\tilde{\triangle}\left[H^{\beta} \boldsymbol{N}\right] \cdot \boldsymbol{N}=-\tilde{\triangle} H^{\beta}-H^{\beta}\left(\boldsymbol{N}_{u u}+\boldsymbol{N}_{v v}\right) \cdot \boldsymbol{N},
\end{aligned}
$$

\footnotetext{
${ }^{1}$ For isolated umbilic points, a proper pair of principal directions can be determined by the shape of its local neighborhood; for spherical umbilic points, any orthonormal basis in the tangent space may be used [13].
}

where $H^{\beta}=2 \beta(u, v) H$. Using the Weingarten equations [13], it can be shown that

$$
\boldsymbol{N}_{u u} \cdot \boldsymbol{N}=-<\boldsymbol{N}_{u}, \boldsymbol{N}_{u}>=-e^{2} E=-e^{2}
$$

and $\boldsymbol{N}_{v v} \cdot \boldsymbol{N}=-g^{2}$. After some math, we have

$$
\boldsymbol{F}_{\text {rigid }}(\boldsymbol{X}(u, v)) \cdot \boldsymbol{N}=-\tilde{\triangle} H^{\beta}+H^{\beta}\left(4 H^{2}-2 K\right) .
$$

To derive its level set expression, we first define $\rho=$ $\tilde{\triangle} H^{\beta}$. Unlike a curve, for surface it is not easy to compute $\rho$ directly. Instead, we will use the fact that the Laplacian operator is invariant under unitary transformations in particular, a change of orthonormal basis. We first extend the function $\beta(u, v)$ from the zero level set onto the 3-D grid so that it is now a function of $\boldsymbol{x}=(x, y, z)^{T}$. Now note that the mean curvature of the level sets of $\phi$ is also a function of $\boldsymbol{x}$. We now have $H^{\beta}$ as a function of $\boldsymbol{x}$. The standard 3-D Laplacian of this function can be easily computed. The directional second derivative of this function along the normal direction of the level set can be computed using

$$
\frac{\partial H^{\beta}}{\partial \boldsymbol{N}}=\frac{\nabla \phi^{T} \boldsymbol{H}\left(H^{\beta}\right) \nabla \phi}{|\nabla \phi|^{2}},
$$

where $\boldsymbol{H}\left(H^{\beta}\right)$ is the Hessian matrix of $H^{\beta}(\boldsymbol{x})$. Thus the Laplacian of $H^{\beta}$ in the zero level parameter space (tangent space) is given by

$$
\rho=\tilde{\triangle} H^{\beta}=\triangle H^{\beta}-\frac{\nabla \phi^{T} \boldsymbol{H}\left(H^{\beta}\right) \nabla \phi}{|\nabla \phi|^{2}} .
$$

The level set expression of Gaussian curvature $K$ is given by [32]

$$
K=\frac{\nabla \phi^{T} \operatorname{Adj}(\boldsymbol{H}(\phi)) \nabla \phi}{|\nabla \phi|^{2}},
$$

where $\boldsymbol{H}(\phi)$ is the Hessian matrix of $\phi(\boldsymbol{x})$, and $\operatorname{Adj}(\boldsymbol{H})$ is the adjoint matrix of $\boldsymbol{H}$.

Thus, the level set expression of the normal component of the rigid force is given by

$$
\boldsymbol{F}_{\text {rigid }} \cdot \boldsymbol{N}=-\rho+H^{\beta}\left(4 H^{2}-2 K\right) .
$$

The external forces in active surfaces can be directly generalized from active contours. Therefore, analogous to active contours, we obtain the following GAS formulation

$$
\begin{aligned}
& \gamma \phi_{t}(\boldsymbol{x})=\left[2 \alpha(\boldsymbol{x}) H+H^{\beta}\left(4 H^{2}-2 K\right)-\rho(\boldsymbol{x})\right]|\nabla \phi(\boldsymbol{x})| \\
& \quad+w_{\text {pres }}(\boldsymbol{x})|\nabla \phi(\boldsymbol{x})|-\boldsymbol{F}_{\text {ext }}(\boldsymbol{x}) \cdot \nabla \phi(\boldsymbol{x}) .
\end{aligned}
$$

\section{Implementation Issues and Remarks}

\subsection{Implementation Issues}

We implemented both the GAC of (10) and the GAS of (22) using the narrow band approach described in [1]. Rebuilding the narrow band when the contour or surface hits the narrow band boundary is calculated using the fast marching algorithm described in $[2,31]$. To assure the stability of the algorithm, the rigid force is computed based on the algorithm proposed by Chopp and Sethian [10]. 


\subsection{Remarks on the Mean Curvature Flow}

We should note that while the geometric heat flow of curves will shrink an arbitrary simple closed curve to a round point without developing singularities, it is well known that the mean curvature flow (active surface evolution with only elastic force and constant $\alpha$ ) may not shrink an arbitrary simple surface to a spherical point without causing singularities, e.g., a dumbbell surface. Several researchers [7, 11, 21, 22] have proposed geometric flows with improved smoothing abilities by incorporating Gaussian curvature $K$. For example, based on Alvarez's axioms [3], Caselles and Sbert [7] considered the flow, $\boldsymbol{X}_{t}=$ $\operatorname{sign}(H)\left(K^{+}\right)^{1 / 4} \boldsymbol{N}, K^{+}=\max (K, 0)$, and proved that this flow smoothly shrinks rotationally symmetric dumbbells to ellipsoidal shaped points without developing singularities. Neskovic and Kimia [21] enlarged the class of surface that can be shrinked to spherical points by considering the flow, $\boldsymbol{X}_{t}=\operatorname{sign}(H) \sqrt{K+|K|} \boldsymbol{N}$. Special geometric active surface models using these flows with improved smoothing ability can be easily obtained from (22) by choosing elastic force's spatially-varying weight $\alpha(x)$ as either $\frac{\operatorname{sign}(H)}{H}\left(K^{+}\right)^{1 / 4}$ or $\frac{\operatorname{sign}(H)}{H} \sqrt{K+|K|}$.

\subsection{Remarks on the Fourth-order Flow}

So far, the geometric 4th-order flow (rigid force) is much less understood than the 2nd-order flow, and is still an active research topic. The following observations on geometric 4th-order flows of curves and surfaces are taken largely from the thesis of Polden [26]. A natural 4th-order analogue of the 2nd-order geometric heat flow $\boldsymbol{X}_{t}=\kappa \boldsymbol{N}$ is given by $\boldsymbol{X}_{t}=-\kappa_{s s} \boldsymbol{N}$, where $\kappa_{s s}$ denotes the second derivative of curvature with respect to the arclength parameter $s$. Note that the inward unit normal $\boldsymbol{N}$ is used here. If the outward normal is used, then $-\kappa$ and $\kappa_{s s}$ should be used instead. While it is well known that the 2nd-order geometric heat flow arises as the gradient flow for length, this 4th-order analogue does not arise directly from a variational problem. This flow, however, does decrease curve length. Furthermore, it keeps the area of the curve fixed and hence improves the isoperimetric ratio. A closely related 4th-order flow comes from minimizing the total squared curvature $\int \kappa^{2} d s$. Such functionals arise in the theory of elasticae. The problem with minimizing this for a simple closed curve is that the elastic energy can be made arbitrarily small by magnifying the curve (rescaling), making the problem illposed. However, by adding a penalty on arclength along with the elastic penalty, the problem becomes well-posed. In this case, the cost functional has the form $\int\left(\kappa^{2}+\alpha\right) d s$, where $\alpha>0$ denotes the weight on the length penalty, and the long-term regularity of the resulting flow is proven in [26] not only for smoothly embedded curves, but for immersed curves (i.e. curves which may self-intersect, such as a figure eight) as well. The corresponding 3-D surface flow would come from minimizing the total squared mean curvature (the Willmore energy) along with a penalty on surface area. It was conjectured by DiGiorgi that this flow

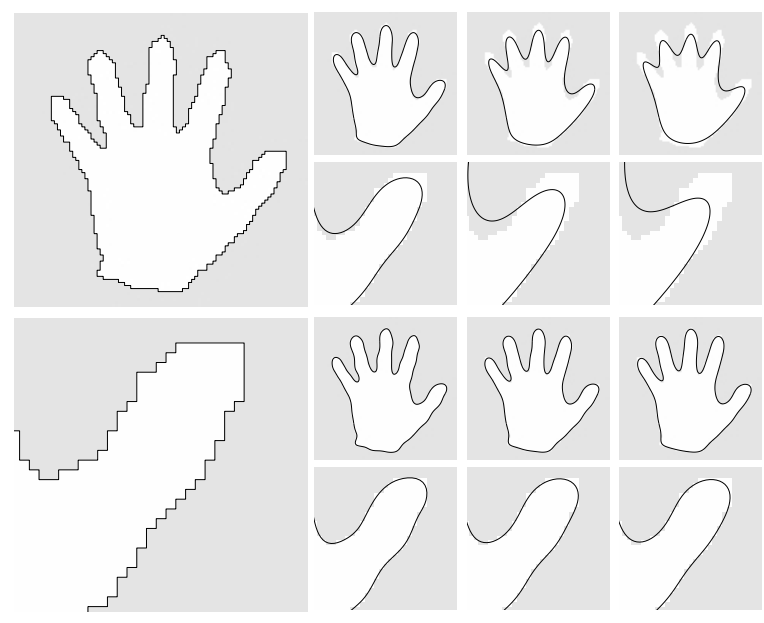

Figure 1. A jagged hand contour (top left) and a zoom up around the thumb (bottom left). Active contour deformed under elastic force with $\alpha=0.1$ (top right) and under rigid force with $\beta=0.1$ (bottom right) at 100 , 500 , and 1000 iterations, respectively.

would convexify any smoothly embedded compact surface in a regular manner, but this is still an open problem as no proof or counter example has yet appeared.

\subsection{Remarks on the Limitations of the Relationship}

The relationship we have derived does not provide a complete equivalence. From the outset we eliminated all terms that depend on an explicit parameterization of the active contour or surface. But a parameterization may be required for certain PACs or PASs. For example, spring forces use springs that are tied to particular points on the contour or surface (cf. [14]). They are Lagrangian in character, and it would require contour or surface point tracking to implement them within an Eulerian framework. Active contours or surfaces that mimic physical objects whose tension and rigidity coefficients varying along the curve likewise cannot be implemented without contour or surface point tracking. So, to complete the equivalence, some way to keep track of the initial parametric coordinate of a contour or surface point, given its corresponding spatial location is required. This is a subject for future research.

\section{Applications}

This section shows the application of the mathematical relationship derived in Section 3 and 4 for designing new GACs based on two recent PACs. The GAS formulations for these applications can be similarly derived using (22), but are omitted here due to lack of space.

Shape fairing. It has sometimes been argued that the active contour rigidity term is not necessary, that the elasticity term is adequate [6]. But the elasticity term shrinks contours, which may be undesirable in some applications. Use 
of the rigidity term (non-zero $\beta$ ) with small or no elasticity can help provide contour smoothness - fairing the contour — while minimizing contour shrinkage. Equation (10) provides a way to do this within the GAC framework. Fig. 1 contrasts the contour smoothing difference between elastic forces and rigid forces.

Region-based forces. Region information, when it could be reliably estimated, e.g., from image segmentation, can be used to improve the robustness of an active contour, both to noise and to weak edges. Those PACs formulations that have incorporated region information - e.g., [8, 27, 28, 44] - can all be written in the following way

$$
\begin{gathered}
\gamma \boldsymbol{X}_{t}=\left[\alpha(s) \boldsymbol{X}_{s}\right]_{s}-\left[\beta(s) \boldsymbol{X}_{s s}\right]_{s s} \\
+w_{R} R(\boldsymbol{X}) \boldsymbol{N}+\boldsymbol{F}_{\text {ext }}(\boldsymbol{X}),
\end{gathered}
$$

where $R(\boldsymbol{x})$ is a region indicator function and $w_{R}$ is a positive weighting parameter. $R(\boldsymbol{x})$ is derived from the image and (for the sake of concreteness) has values in the range $[-1,1]$ that are smaller within the region(s)-of-interest. It modulates the sign of the pressure forces using region information so that the contour shrinks when it is outside the object of interest and expands when it is inside the object. For this reason, these external forces are sometimes called signed pressure forces.

Signed pressure forces help to solve the so-called boundary leaking problem, which results from weak edges. This idea has only recently been incorporated into GACs $[9,24$, $25,29,42,43$, and our equivalence relationship permits a direct and more general result. In particular, using (9) and (10), we can easily write

$$
\begin{gathered}
\gamma \phi_{t}(\boldsymbol{x})=\left[\alpha(\boldsymbol{x}) \kappa(\boldsymbol{x})+\beta(\boldsymbol{x}) \kappa^{3}(\boldsymbol{x})-\rho(\boldsymbol{x})\right]|\nabla \phi(\boldsymbol{x})| \\
+w_{R} R(\boldsymbol{x})|\nabla \phi(\boldsymbol{x})|-\boldsymbol{F}_{\text {ext }}(\boldsymbol{x}) \cdot \nabla \phi(\boldsymbol{x})
\end{gathered}
$$

which comprises a more general class of region-based, GACs than has previously been reported.

Gradient vector flow forces. Active contours using gradient vector flow (GVF) external forces have been shown to have a larger capture range and the ability to converge into boundary concavities $[38,39]$. They have been used only in PAC formulations, however, because they comprise non-conservative forces that do not fit within the standard geometric (or geodesic) active contour framework. Using our derived equivalence, it is straightforward to develop a GVF GAC.

A GVF field is defined as the equilibrium solution of a generalized vector diffusion equation

$$
\boldsymbol{v}_{t}=g(|\nabla f|) \nabla^{2} \boldsymbol{v}-h(|\nabla f|)(\boldsymbol{v}-\nabla f),
$$

where $\boldsymbol{v}(\boldsymbol{x}, 0)=\nabla f, \boldsymbol{v}_{t}$ denotes the partial derivative of $\boldsymbol{v}(\boldsymbol{x}, t)$ with respect to $t, \nabla^{2}$ is the Laplacian operator (applied to each spatial component of $\boldsymbol{v}$ separately), and $f$ is an edge map that has a higher value at the desired object boundary. The functions $g(r)$ and $h(r)$ control the amount of diffusion in GVF; in this paper, we use

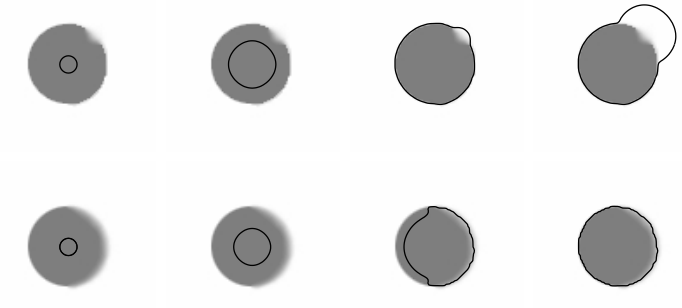

Figure 2. Result from the previous GAC (8) (top row) and the proposed region-based GAC (bottom row).

$g(r)=\exp \left\{-(r / K)^{2}\right\}$ and $h(r)=1-g(r)$, where $K$ is a scalar controlling the tradeoff between field smoothness and gradient conformity. We define $\tilde{\boldsymbol{v}}=\boldsymbol{v}(\boldsymbol{x}, \infty)$, the equilibrium state of (25). A general GVF GAC follows by substituting $\tilde{\boldsymbol{v}}(\boldsymbol{x})$ for $\boldsymbol{F}_{\text {ext }}(\boldsymbol{x})$ in (24), yielding

$$
\begin{aligned}
\gamma \phi_{t}(\boldsymbol{x}) & =\left[\alpha(\boldsymbol{x}) \kappa(\boldsymbol{x})+\beta(\boldsymbol{x}) \kappa^{3}(\boldsymbol{x})-\rho(\boldsymbol{x})\right]|\nabla \phi(\boldsymbol{x})| \\
& +w_{R} R(\boldsymbol{x})|\nabla \phi(\boldsymbol{x})|-\tilde{\boldsymbol{v}}(\boldsymbol{x}) \cdot \nabla \phi(\boldsymbol{x})
\end{aligned}
$$

If desired or necessary, region forces can be turned off by setting $w_{R}=0$.

Volcano forces. Volcano forces are local repulsive forces that are determined by a volcano location specified by the user [14]. They are a powerful tool for interactive snake manipulation commonly used in parametric snakes. They are readily implemented as static external forces and either add to or replace $F_{\text {ext }}(\boldsymbol{x})$.

\subsection{Examples}

Figure 2 shows an example patterned after one in [33], in which there is a weak boundary at the top right of the circle. The top row of this figure shows a conventional GAC leaking through this boundary, while the bottom row shows a region-based GAC implemented using (24). To construct the region indicator function in this example, we applied fuzzy C-means to automatically classify the figure into two classes, each with a fuzzy membership value at each pixel. The region indicator function was then given by

$$
R(\boldsymbol{x})=1-2 \mu_{f}(\boldsymbol{x})
$$

where $\mu_{f}(\boldsymbol{x})$ is the membership function corresponding to the darker intensity class. To better localize the boundaries, these experiments also used an additional external force given by

$$
\boldsymbol{F}_{\text {ext }}(\boldsymbol{x})=\nabla \mu_{f}(\boldsymbol{x}) .
$$

Fig. 3 shows the results of applying our region-based GAC to segment the brain ventricles and the white matter from an MR image. The only difference between the results shown in the top row and the second row is in the definition of the region indicator function, which was based on the 

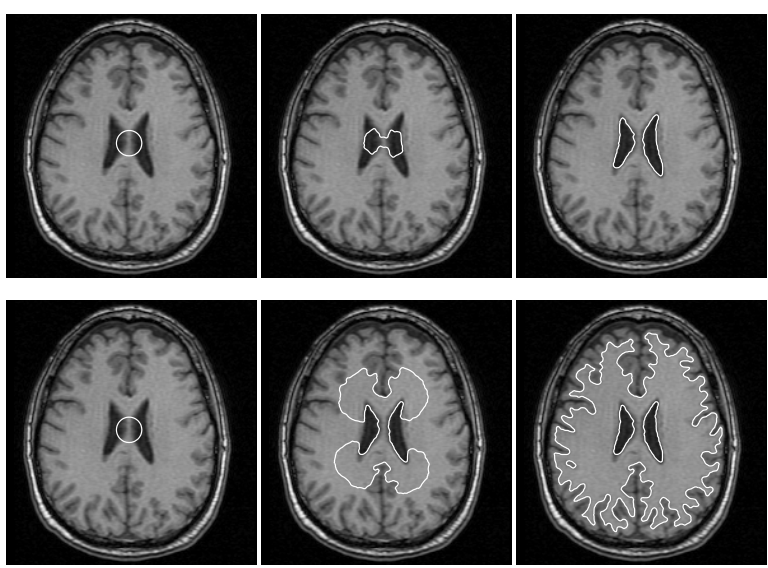

Figure 3. A region-based GAC can extract either the ventricle (top row) or the white matter (bottom row).

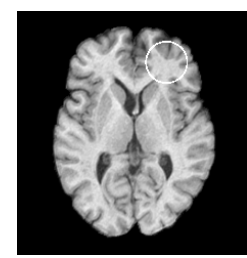

(a)

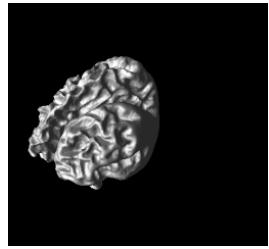

(d)

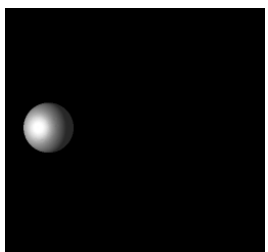

(b)

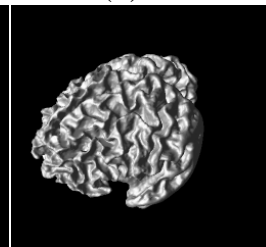

(e)

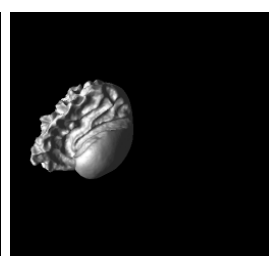

(c)

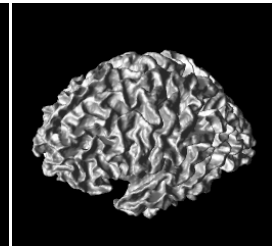

(f)
Figure 4. An example of a region-based GAS. (a)-(b) Surface initialized as a sphere across the white matter boundary. (c)-(f) Intermediate deformations and the final extracted white matter surface.

ventricle membership (top row) and the white matter membership (bottom row), respectively. Fig. 4 shows a result of applying our region-based GAS for volumetric brain MR image segmentation. An initial sphere is initialized across the brain white matter boundary (see Fig. 4(a) for its initial position in a cross-sectional image), and allowed to evolve using the GAS. The sphere shrinks at places where it is outside white-matter, expands where it is in white-matter, and eventually recovers a complete 3 -D surface representation of the white matter boundary.

In applications where reliable region information is difficult to estimate and there exist boundary gaps, GVF GACs could provide an useful alternative, since they preserve the perceptual edge property of active contours $[14,39]$. Fig. 5 shows an example in which a GVF GAC was applied to a simulated image containing an object that has both boundary gaps and concavities. In Fig. 5(a), a contour is initial-

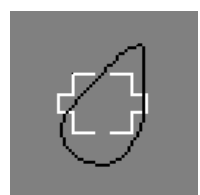

(a)

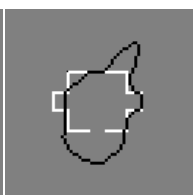

(b)

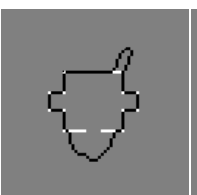

(c)

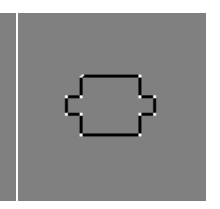

(d)
Figure 5. A GVF GAC is initialized across the object boundary in a simulated image (a) and deforms to the desired shape by simultaneously detecting boundary concavities and bridging boundary gaps (b)-(d).

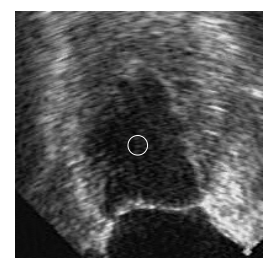

(a)

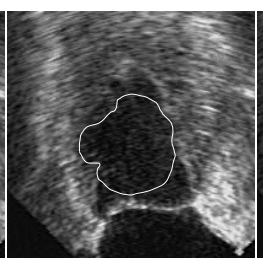

(b)

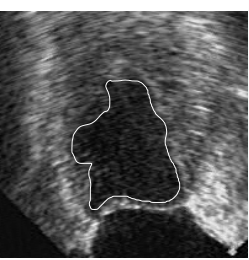

(c)
Figure 6. Segmentation of the LV on a cardiac ultrasound image from a single GVF GAC.

ized across the object boundary. Figs. 5(b) and 5(c) plot the contour deforming towards the desired object boundary. The final result shown in Fig. 5(d) demonstrates the ability of the GVF GAC to recover the boundary reliably in the presence of both boundary concavities and gaps.

Figure 6 shows a GVF GAC applied to a noisy ultrasound image of the left ventricle. We note that the standard geodesic active contour of [6] or [41] failed to produce the correct result on this image.

\section{Summary and Conclusion}

We have derived an explicit mathematical relationship between the general formulations of parametric and geometric active contours and surfaces. This relationship highlights the Lagrangian nature of parametric active contours and surfaces and the Eulerian nature of geometric active contours and surfaces. Of particular note is the inclusion of rigidity in this equivalence. Described applications of the relationship include curve and surface fairing, region-based and gradient vector flow active contours and surfaces, and interactive volcano forces. Examples on simulated and real images were presented, demonstrating in particular its effectiveness on the boundary leaking problem. We expect that the equivalence derived here will facilitate the future development of active contours and surfaces.

\section{Acknowledgments}

The authors thank Xiao Han for his assistance in implementing the narrow-band method for level set evolution and Dzung Pham for providing the fuzzy c-means algorithm. The work was supported in part by an NSF/ERC grant CISST-9731748 and NIH/NINDS grant R01NS37747. 


\section{References}

[1] D. Adalsteinsson and J. A. Sethian. A fast level set method for propagating interfaces. J. Comp. Phys., 118:269-277, 1995.

[2] - . The fast construction of extension velocities in level set methods. J. Comp. Phys., 148:2-22, 1999.

[3] L. Alvarez et al. Axioms and fundamental equations of image processing. Arch. Rational Mech. Anal., 123:199-257, 1993.

[4] G. Aubert and L. Blanc-Féraud. Some remarks on the equivalence between 2D and 3D classical snakes and geodesic active contours. IJCV, 34:19-28, 1999.

[5] V. Caselles et al. A geometric model for active contours. Numerische Mathematik, 66:1-31, 1993.

[6] V. Caselles, R. Kimmel, and G. Sapiro. Geodesic active contours. IJCV, 22:61-79, 1997.

[7] V. Caselles and C. Sbert. What is the best causal scale space for 3D images? Univ. Illes Balears, Palma de Mallorca, Spain, Tech. Rep., 1994.

[8] A. Chakraborty, L. H. Staib, and J. S. Duncan. Deformable boundary finding in medical images by integrating gradient and region information. IEEE-TMI, 15(6):859-870, 1996.

[9] T. Chan and L. Vese. An active contour model without edges. Int. Conf. Scale-Space Theories Comp. Vis., (Lect. Notes Comp. Sci.), 1682:141-151, 1999.

[10] D. Chopp and J. A. Sethian. Motion by intrinsic laplacian of curvature. Interfaces \& Free Boundaries, 1:107-123, 1999.

[11] B. Chow. Deforming convex hypersurfaces by the $n$th root of the Gaussian curvature. J. Diff. Geom., 22:117-138, 1985.

[12] L. D. Cohen. On active contour models and balloons. CVGIP-IU, 53(2):211-218, 1991.

[13] M. P. do Carmo. Differential Geometry of Curves and Surfaces. Prentice-Hall, Englewood Cliffs, NJ, 1976.

[14] M. Kass, A. Witkin, and D. Terzopoulos. Snakes: active contour models. IJCV , 1:321-331, 1987.

[15] S. Kichenassamy et al. Conformal curvature flows: from phase transitions to active vision. Arch. Rational Mech. Anal., 134:275-301, 1996.

[16] B. B. Kimia, A. R. Tannenbaum, and S. W. Zucker. Shapes, shocks, and deformations I: the components of twodimensional shape and the reaction-diffusion space. IJCV, 15:189-224, 1995.

[17] R. Malladi, J. A. Sethian, and B. C. Vemuri. Shape modeling with front propagation: a level set approach. IEEE-TPAMI, 17:158-175, 1995.

[18] T. McInerney and D. Terzopoulos. Topologically adaptable snakes. ICCV, 840-845, 1995.

[19] — Deformable models in medical image analysis: a survey. Med. Imag. Anal., 1:91-108, 1996.

[20] —. Topology adaptive deformable surfaces for medical image volume segmentation. IEEE-TMI, 18:840-850, 1999.

[21] P. Neskovic and B. B. Kimia. Three-dimensional shape representation from curvature dependent evolution. ICIP, 6-10, 1994.

[22] P. J. Olver, G. Sapiro, and A. Tannenbaum. Invariant geometric evolutions of surfaces and volumetric smoothing. $J$. Diff. Geom., 22:117-138, 1985.
[23] S. Osher and J. A. Sethian. Fronts propagating with curvature-dependent speed: algorithms based on HamiltonJacobi formulations. J. Comp. Physics, 79:12-49, 1988.

[24] N. Paragios and R. Deriche. Geodesic active contours and level sets for the detection and tracking of moving objects. IEEE-TPAMI, 22:1-15, 2000.

[25] —. Unifying boundary and region-based information for geodesic active tracking. CVPR, 300-305, 1999.

[26] A. Polden. Curves and surfaces of least total curvature and fourth-order flows. PhD Thesis, Universitat Tubingen, 1996.

[27] C. S. Poon and M. Braun. Image segmentation by a deformable contour model incorporating region analysis. Phys. Med. Biol., 42:1833-1841, 1997.

[28] R. Ronfard. Region-based strategies for active contour models. IJCV , 13:229-251, 1994.

[29] C. Samson et al. A level set method for image classification. Int. Conf. Scale-Space Theories Comp. Vis., (Lect. Notes Comp. Sci.), 1682:306-317, 1999.

[30] G. Sapiro and A. Tannenbaum. Affine invariant scale-space. IJCV , 11:25-44, 1993.

[31] J. A. Sethian. A fast marching level set method for monotonically advancing fronts. Proc. Nat. Acad. Sci., 93:1591-1595, 1996.

[32] —. Level Set Methods and Fast Marching Methods. Cambridge University Press, Cambridge, UK, 2nd edition, 1999.

[33] K. Siddiqi et al. Area and length minimizing flows for shape segmentation. IEEE-TIP, 7:433-443, 1998.

[34] L. H. Staib and J. S. Duncan. Boundary finding with parametrically deformable models. IEEE-TPAMI, 14:10611075, 1992.

[35] D. Terzopoulos. Regularization of inverse visual problems involving discontinuities. IEEE-TPAMI, 8:413-424, 1986.

[36] R. Whitaker and D. Chen. Embedded active surfaces for volume visualization. ECRC-94-8, European ComputerIndustry Research Centre GmbH, 1994.

[37] C. Xu, D. L. Pham, and J. L. Prince. Image segmentation using deformable models. In Handbook of Med. Imag.: Vol. 2. Med. Image Proc. \& Anal., SPIE Press, 2000.

[38] C. Xu and J. L. Prince. Generalized gradient vector flow external forces for active contours. Signal Processing, 71:131139, 1998.

[39] - . Snakes, shapes, and gradient vector flow. IEEE-TIP, 7:359-369, 1998.

[40] C. Xu, A. Yezzi, and J. L. Prince. On the relationship between parametric and geometric active contours. In Proc. of Asilomar Conf. on Signals, Systems. \& Computers, 2000.

[41] A. Yezzi et al. A geometric snake model for segmentation of medical imagery. IEEE-TMI, 16:199-209, 1997.

[42] A. Yezzi, A. Tsai, and A. Willsky. A fully global approach to image segmentation via coupled curve evolution equations. To appear in J. Visual Communication \& Image Repr.

[43] - . A statistical approach to image segmentation for biomodal and trimodal imagery. ICCV, 898-903, 1999.

[44] S. Zhu and A. Yuille. Region competition: Unifying snakes, region growing, and Bayes/MDL for multiband image segmentation. IEEE-TPAMI, 18:884-900, 1996. 